\section{A new probe technique for the identification of serial learning processes*}

\author{
CARLA J. POSNANSKY and WILLIAM F. BATTIG \\ University of Colorado, Boulder, Colorado 80302 \\ and \\ JAMES F. VOSS \\ University of Pittsburgh, Pittsburgh, Pennsylvania 15213
}

We describe a new serial learning probe technique which permits identification of functional stimuli for each individual item immediately following the trial on which it has been learned. Some empirical evidence indicating the validity of the serial probe technique is also presented. The potential superiority of the probe technique over alternative experimental methods for determining the basic processes involved in serial learning is noted.

Serial learning (SL) traditionally has been conceptualized within the verbal learning literature in one of two fundamental ways. These are (1) the acquisition of a chain of responses wherein each item is the stimulus for the succeeding item, and (2) the acquisition of a set of associations between items and their serial positions wherein each serial position is the stimulus for the corresponding item. Combinations of these two basic conceptions have comprised several hypotheses with regard to the functional stimulus in SL, and comprehensive reviews of recent research testing these hypotheses have been provided by Young (1968) and Battig (1969).

It should be emphasized, however, that transfer tasks introduced only after completion of learning of serial lists by an anticipation method have comprised the primary methodology for the research in the area thus far and that such a methodology necessarily contains important limitations. First, with regard to the use of a transfer task, after completion of List 1 learning, it should be acknowledged that such tasks are likely to be contaminated by some type of interference which counteracts any positive transfer occurring and, therefore, obscures any process or functional stimuli involved during SL. Also, as noted by previous researchers (e.g., Postman, 1968; Voss, 1968), the utilization of a transfer task does not allow a distinction to be made between the manner in which the serial list was learned and the manner in which knowledge about the serial list may be used during the transfer task. Furthermore, the use of a

* This research was largely supported by the National Science Foundation through a predoctoral fellowship held by the first author and Grant GB-25433 to the second author. This is Publication No. 18 of the Institute for the Study of Intellectual Behavior, University of Colorado. subsequent transfer task necessarily involves attempted identification of the functional stimulus for various items in the serial list after different stages of learning. That is, each item in a given list may actually have been learned on a different trial, although all are tested only after the entire list has been learned to criterion. Consequently, any changes that occur in the functional stimulus for a given item with increased learning may be obscured by a methodology which does not measure the degree of learning for each item. To this point, Voss (1969) was able to demonstrate that the learning of items in a serial list and placement of these items in their
PRIOR ITEM CUE

POSITION CUE

3 PRIOR ITEM CUE proper serial positions occurred simultaneously for items at the beginning of the list. At the later positions, however, items first were learned and subsequently placed in their proper serial positions. This demonstration, that the degree of learning did affect the identification of a functional stimulus, was made by using three types of interruption tasks (free recall, anticipation, or responses to an item's serial position as designated by a number) at various stages of learning the serial list.

The methodology described in the present paper is an extension of this general approach, representing a technique that allows identification of the functional stimulus in SL for items of a known and equivalent stage of learning. Additionally, the present procedure utilizes a version of the serial recall method rather than the serial anticipation method for studying SL in order to permit identification of the functional stimulus after an item is given correctly for the first time without allowing any additional exposure to the specific item.

The present report includes a general description both of the probe technique as it has been used thus far and of the empirical evidence resulting from its use in a specific instance.

GENERAL PROCEDURAL DESCRIP'TION

Utilizing a variation of the serial
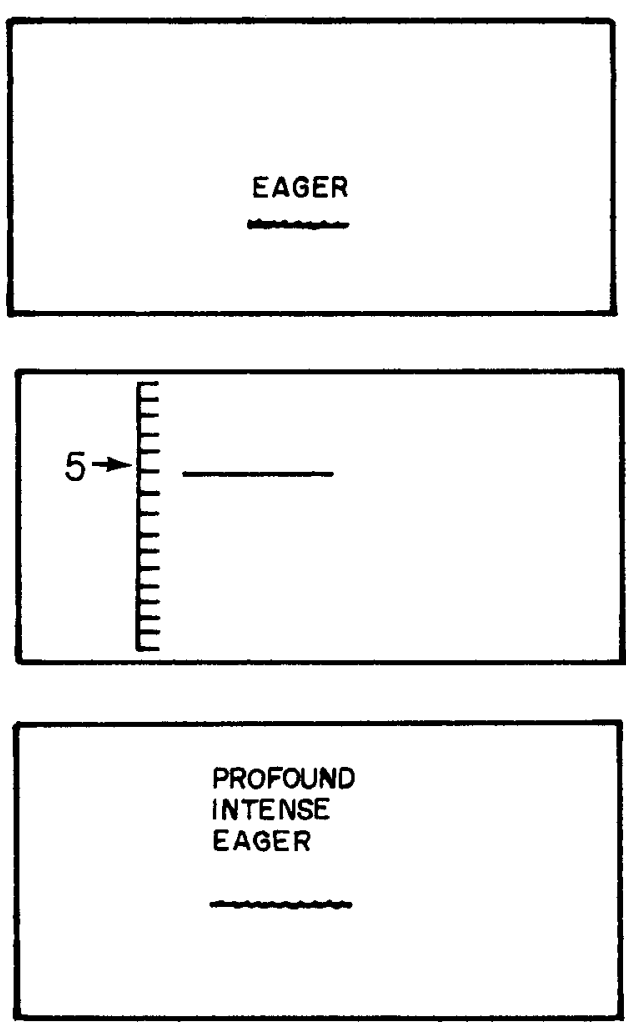

Fig. 1. Examples of probe types. 
Table 1

Probe Sequences

\begin{tabular}{ccllc}
\hline $\begin{array}{l}\text { Type of } \\
\text { Sequence }\end{array}$ & $\begin{array}{c}\text { Number } \\
\text { of Items }\end{array}$ & $\begin{array}{l}\text { First } \\
\text { Cue }\end{array}$ & $\begin{array}{c}\text { Second } \\
\text { Cue }\end{array}$ & $\begin{array}{c}\text { Third } \\
\text { Cue }\end{array}$ \\
\hline 1 & 6 & POS & POS + PI & POS + 3PI \\
2 & 3 & PI & POS + PI & POS + 3PI \\
3 & 3 & PI & 3PI & POS + 3PI \\
\hline
\end{tabular}

Key-POS denotes position cue, PI denotes prior item cue, and $3 P I$ denotes three prior item cue.

recall method of SL, the Ss were asked to view each item in the serial list on a memory drum tape for $2 \mathrm{sec}$ and then, after the last item had been presented, Ss were given an unlimited time in which to recall the words in the list. Instructions indicated that if at any time S knew he was "skipping" an item in the list $\mathbf{S}$ should represent the nonremembered item with the word "SKIP." Thus, an item was to be scored as "correct" if and only if it was given correctly in the proper serial position.

Immediately after each recall trial, a probe technique was employed in order to ascertain directly the nature of the functional stimulus for individual items within the serial list. This probe technique is similar to that developed by Murdock and others for probing short-term memory (e.g., Woodward \& Murdock, 1968). Under this procedure, either a diagrammatic representation of the item's serial position or, alternatively, its immediately preceding item was presented to $S$ on an index card. Examples of the probe cards can be seen in Fig. 1. After the card presentation, $S$ was given $5 \mathrm{sec}$ in which to respond with the correct item. If $S$ could not do so, an additional card was presented on which either the alternative cue or a new cue of the three preceding items was added to the original cue. After another $5 \mathrm{sec}$ period, if no correct response had yet been given, the third cue was added to the first two cues. The sequence of probe types and the first probe responded to correctly were recorded for all probed items in the list, the sequence of probe types being counterbalanced across items This essentially meant that Ss received the prior-item cue first for half the items in the list and the serial-position cue first for the other half of the items. A delineation of the probe sequences is seen in Table 1 . Using a varied sequence of probe schemes on different items presented the additional advantage of minimizing any bias toward or against any particular process(es) of serial learning.

Instructions to $S s$ regarding the occurrence of probes were given with the SL instructions prior to SL. Instructions explained the use of the probe cards, the time intervals involved, and the oral responding required. These instructions did not, however, indicate the contingencies of probing, and the three probe types used were not specifically illustrated.

Within the present use of the probe technique, probes were applied to all but the first three items in the serial list, since no three-prior-item cue was available for any of these first three items. However, this is not a necessary limitation of the probe technique, since it is quite possible to apply other types of probes to these beginning items.

The application of the probe to particular items within the list after a given recall trial remained contingent upon S's performance on a given recall trial. That is, in order to identify the functional stimulus for an item just learned, the probe procedure was applied to a given item immediately after the trial on which that item was given correctly for the first time. It is possible, of course, to probe any given item at other stages of learning as well although it is doubtful that repeated probing of the same item on more than one trial will yield any useful additional information.

Additionally, this procedure allowed charting of changes in an item's functional stimulus with increased learning, for different Ss could receive probes after different degrees of learning. Thus, the stage of learning for a given item was precisely controlled and, since each item was probed individually, this procedure also permitted an analysis of the effect of an item's serial position upon its functional stimulus.

In order to permit evaluation of the effectiveness of the probes, a control procedure was employed in which probes were given which were not contingent upon S's performance on a given trial. Thus, the items to be probed on a given trial were previously and randomly selected, so it was possible to study the effectiveness of probes for items not yet "learned." Probes were expected to be ineffective for these items, except to the extent that the stringent scoring criterion permitted something about an item (e.g., its prior item) to be learned before the item was counted as correct. To maintain equivalence with the contingent probe conditions, it was considered desirable to probe a number of items on each trial equal to the number first recalled correctly on that trial.

Additionally, the present technique permitted evidence regarding the possible existence of more complicated functional stimuli or SL processes to be collected. This is indexed by the overall effectiveness of the probes for learned items. That is, a large frequency of failures to respond to any of the various probes would strongly suggest that more complex processes are of primary importance in the SL task.

\section{EMPIRICAL EVIDENCE}

Subjects

The $60 \mathrm{Ss}$ in the present study were introductory psychology students at the University of Colorado participating in order to fulfill a course requirement.

\section{Materials}

A 15-item serial list composed of adjectives was constructed from Melton and Safier's list as cited in Hakes, James, and Young (1964). Formal and meaningful similarity were kept minimal. Two different serial orders of these 15 items were used, each for half of the Ss in each group.

\section{Procedure}

The procedure and probing technique previously described were utilized in the present study for two experimental groups of 24 Ss each and one control group of 12 Ss.' Within the experimental first-correct group, Ss received probes for items recalled correctly for the first time on a given trial. For the third-correct experimental group, however, items were probed after they were recalled for the third time. In the control group, items were probed in the noncontingent manner described previously. Trials were continued until each item had been recalled correctly once (for the control and experimental first-correct groups) or three times (for the experimental third-correct group).

\section{Results}

The number of times a given type of probe (prior item or serial position) was responded to correctly as a first probe or as an added probe across a pair of serial positions (fourth and fifth positions, sixth and seventh positions, etc.) was tabulated for each $S$ for each of the six pairs of probed positions. Correct responses to the added three-prior-item cue were so infrequent (less than 13\%) that no meaningful tabulations or analyses were possible. 

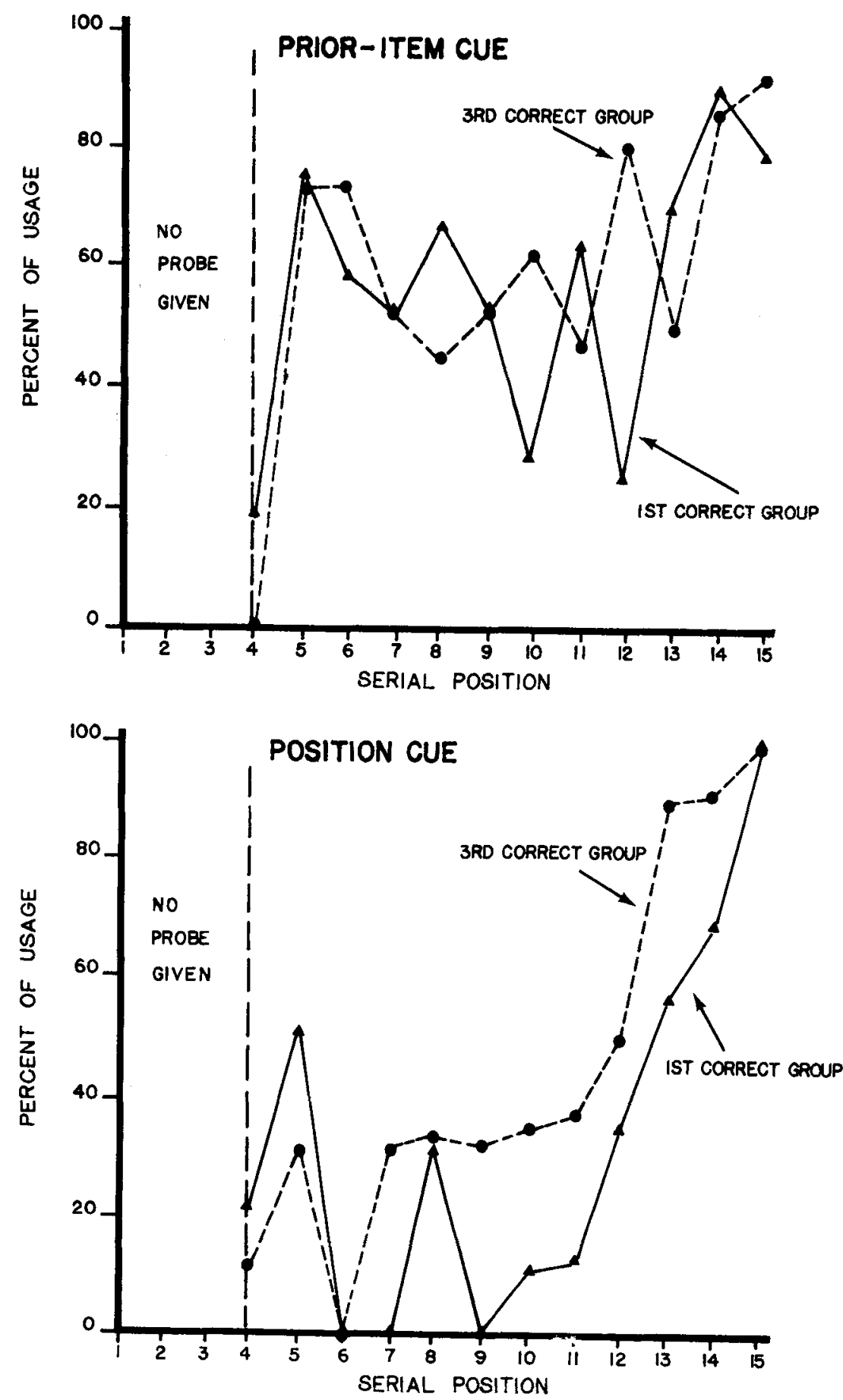

Fig. 2. Percentage of total opportunities for each serial position where the initial presentation of the prior item cue (top graph) and of the position cue (bottom graph) elicited the correct response.

Separate analyses of variance for each probe type indicated that usage of the preceding item cue did not differ between the two experimental groups, despite their differing degrees of learning preceding the probes. The serial position cue was, however, used significantly more by the third-correct than by the first-correct experimental group, $F(1,44)=5.04, p<.05$.

Usage of both preceding item and position cues differed significantly as a function of serial position, although the serial position effect was substantially greater for the position cue, $F(5,220)=14.39$, $p<.001$, than for the preceding item cue, $F(5,220)=$ $3.26, p<.01$. There was no interaction, however, between the serial position and degree of learning variables.

Figure 2 represents another way of looking at serial position effects, representing the total number of times either the serial position cue or the preceding item cue was effectively used as a first cue. This figure suggests that within the center section of the list the serial position cue may have been more functional for Item 8 (the exact center of the list) than for other middle items. However, this occurred only within the first correct group and failed to replicate in a subsequent experiment (Posnansky, 1971). Otherwise, the results shown in Fig. 2 are consistent with the previous statistical analysis based upon the effective probe occurring anywhere within the probe sequence. It should also be noted that both probe types (but especially the position cue) were least effective in the middle of the list.

The effectiveness of all probes was greatly reduced to $36.5 \%$ correct within the control group in the instances in which items were probed before they were "learned." However, the criterion for a "learned" item was rather stringent in that items were counted as "learned" if and only if they were given correctly in the correct serial position. Thus, it is conceivable that much was actually known about an item (e.g., its prior item) before it was counted as "learned," so that any correct responding to a probe for an "unlearned" item was an artifact of the scoring criterion.

\section{COMMENTS}

The results of the present study demonstrate the value of evaluating the functional stimulus of the SL task within the task, per se, without recourse to a transfer task after completion of SL. The ability to equate degree of learning for individual items, rather than for the list as a whole, has revealed that the effective stimulus depends upon the serial position of the item. Also, the serial position cue was affected by the degree of learning manipulation, while the prior item cue was not.

The serial probe technique has also permitted the evaluation of the functional stimulus for more than one item within each $S$. To this point, under conditions of appropriate counterbalancing wherein each probe sequence occurred equally often for each item across Ss, Posnansky (1971) found that frequency of probe usage was not affected by the number of previously occurring probes, all Fs $<1$. This supports the contention that probing does not significantly influence SL processes. Furthermore, in the present study the presentation of unlearned prior item as a probe did not appear to contribute significantly to $\mathrm{SL}$, since such instances occurred only on the average of 1.6 times per $S$, 
thus providing further evidence that multiple probing within Ss can be done without contaminating side effects.

An additional unpublished pilot study by the authors has lent additional validity to the probe technique by demonstrating that the time intervals taken up by the probes between SL trials, whether or not filled with probes, did not influence the rate of SL. Therefore, the probe technique appears to provide a valuable and relatively uncontaminated general tool for evaluating SL processes when intralist variables are manipulated in the serial list, as well as providing a tool for comparing the processes occurring within the two different methods of serial anticipation and recall. Research in progress (Posnansky, 1971) makes use of the probe technique for such a comparison, as well as for evaluating the effect of an intralist variable (grouping items within the list during learning) upon SL processes.

\section{REFERENCES}

BATTIG, W. F. Sequential learning and thought. In J. F. Voss (Ed.), Approaches to thought, Columbus, Ohio: Merrill 1969.

HAKES, D. T., JAMES, C. T., \& YOUNG R. K. A re-examination of the Ebbinghaus derived-list paradigm. Journal of Experimental Psychology, 1964, 68, 508-514.

POSNANSKY, C. J. Probing for the functional stimuli in serial learning. U npublished Master's thesis, University of Colorado, 1971

POSTMAN, L. Association and performance in the analysis of verbal learning. In $T$. $R$. Dixon and D. L. Horton (Eds.), Verbal behavior and general behavior theory. Englew ood Cliffs, N.J: Prentice-Hall, 1968.

VOSS, J. F. Serial acquisition as a function of number of successively occurring list items. Journal of Experimental Psy chology, 1968, 78, 45 6-462.

VOSS, J. F. Serial acquisition as a function of stage of learning. Journal of Experimental Psychology, 1969, 79, 220-225.

WOODWARD, A. E. JR \& MURDOCK, B. B., JR. Positional and sequential probes in serial learning. Canadian Journal of Psychology, 1968, 22, 131-138.

YOUNG, $R$. $K$. Serial learning. In $T, R$ Dixon and D. L. Horton (Eds.), Verbal behavior and general behavior theory. Englewood Cliffs, N.J: Prentice-Hall, 1968. 